\title{
El Papel Fundamental de la Biología Activista y las Interacciones entre el Museo Nacional, Política y Construcción de Nación en Brasil
}

Edwin de Jesús Bedoya-Roqueme ${ }^{1}$

\section{Book Review:}

Regina Horta Duarte, Activist Biology: The National Museum, Politics, and Nation Building in Brazil (Tucson, Arizona: University of Arizona Pres, 2016).

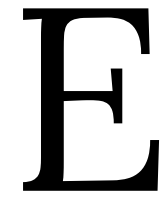
sta reseña se basa en la obra traducida al inglés titulada "Activist Biology: The National Museum, Politics, and Nation Building in Brazil" del libro escrito por Regina H. Duarte titulado "A Biologia Militante: o Museu Nacional, especialização científica, divulgação do conhecimento e práticas políticas no Brasil, 1926-1945", la versión en inglés presenta nuevas fuentes primarias y secundarias, se enriquece la discusión y se incrementan informaciones de diferentes contextos en el campo político, histórico y social, especialmente para el Museo Nacional, la Primera República y la época de Vargas (1930-1945). También se adiciona una línea de tiempo y

\footnotetext{
1 Programa de Pós-graduação em Recursos Naturais do Cerrado, RENAC, Universidade Estadual de Goiás, Brasil. Grupo de Investigación en Biodiversidad Marina y Costera: BIODIMARC. Grupo de estudio de Aracnología PALPATORES. Universidad de Córdoba, Montería, Colombia. ORCID: https://orcid.org/0000-0001-7419-3886. roquemeedj@gmail.com
} 
un marco histórico, en este sentido, se mejoró el significado y la redacción de algunos párrafos, lo cual, facilitó la lectura y comprensión de los diferentes conceptos e ideas presentadas; asimismo, algunos términos y expresiones en portugués fueron reemplazados por expresiones clásicas en inglés, lo cual, facilitó el acceso de los lectores al texto. Con base en lo anterior, el libro presenta explicaciones de algunos términos y conceptos del portugués brasileño, utilizando el mismo estilo de redacción que la autora, todos estos cambios fueron revisados y acompañados por la autora y expresados en la nota del traductor.

Regina Horta Duarte es licenciada en Historia de la Universidad de Minas Gerais, finalizó su curso en 1985, además, hizo una maestría (1988) y doctorado en (1993), ambos en historia de la Universidad Estatal de Campinas ${ }^{2}$. Actualmente ocupa el cargo de Profesora Titular en la Universidad Federal de Minas Gerais y tiene experiencia en Historia, con énfasis en Historia del Imperio de Brasil y República, historia y naturaleza e historia de la biología en la Primera República. Regina fue miembro de la Asociación Nacional de Historia entre los años 2007 y 2009, donde se desempeñó como editora a cargo de la Revista Brasileira de Historia. Fue uno de los miembros fundadores de la Sociedad de Historia Ambiental de América Latina y el Caribe, editora en jefe de la revista Historia Ambiental de América Latina y el Caribe $(\text { HALAC })^{3}$ y es investigadora del nivel 1A del Consejo Nacional de Desarrollo Científico y Tecnológico-CNPq.

Además de artículos en revistas especializadas, Regina ha publicado libros como: "A imagem Rebelde" (1991), "Noites Circenses: espetáculos de circo e teatro em Minas Gerais no século XIX"5 (1995), "A Biologia Militante: o Museu Nacional, especialização científica, divulgação do conhecimento e práticas políticas no Brasil, 1926-1945"6 (2010)7 y "Activist Biology: The National Museum, Politics, and Nation

\footnotetext{
2 UFRJ-Instituto de História, "Regina Horta Duarte, Biografia," Simpósio Diálogo Brasil-EUA em História Ambiental, 2013, http://labhe.historia.ufrj.br/.

${ }^{3}$ Regina Horta Duarte, Activist Biology: The National Museum, Politics, and Nation Building in Brazil (Tucson, Arizona: University of Arizona Pres, 2016).

${ }^{4}$ Regina Horta Duarte, A Imagem Rebelde: A Trajetória Libertária de Avelino Fóscolo (São Paulo: Editora da Universidade Estadual de Campinas, 1991).

${ }^{5}$ Regina Horta Duarte, Noites Circenses: Espetáculos de Circo e Teatro Em Minas Gerais No Século XIX (São Paulo: Editora da Unicamp, 1995).

${ }^{6}$ Regina Horta Duarte, A Biologia Militante: O Museu Nacional, Especialização Científica, Divulgação Do Conhecimento e Práticas Políticas No Brasil, 1926-1945 (Belo Horizonte: Editora UFMG, 2010).
} 
Building in Brazil"8 (2016). En este último, la autora utilizó enfoques de la historia ambiental e informa aspectos muy importantes para el desarrollo histórico y científico de Brasil, como el establecimiento del Museo Nacional de Río de Janeiro y los científicos que desempeñaron un papel clave para la difusión científica y la colaboración en esa época ${ }^{9}$. Entre los años 1985 y 2019, Regina H. Duarte contribuyó en gran medida al conocimiento científico sobre la historia de Brasil, cuya producción científica durante este período incluye 13 libros completos, 48 artículos, 27 capítulos de libros, 20 textos publicados en revistas, 15 artículos publicados en eventos y 42 obras clasificadas como "otros tipos de producción"10.

El libro "Activist Biology: The National Museum, Politics, and Nation Building in Brazil" ofrece una lectura fluida, fácil de seguir y fácil de leer, cuya historia se centra en el tiempo de la República de Brasil durante el gobierno de Getúlio Vargas (19301945) y cómo la ciencia se desarrolló en esa época. El libro es el resultado de una investigación exhaustiva e histórica sobre temas selectivos que involucran diferentes temas de interés para los años en que se desarrolla el relato histórico, como la agricultura, la creación del Museo Nacional y cómo esta institución hizo posible el desarrollo de proyectos sobre ciencia y protección de la naturaleza en Brasil, el establecimiento de las primeras colecciones, los personajes más importantes de la época con énfasis en las trayectorias de Cândido Firmino de Melo Leitão (1886-1948) Zoólogo brasileiro considerado el fundador de Aracnología en Sudamérica, Alberto José de Sampaio (1881-1946), Botánico, uno de los mayores conocedores de la flora brasileira y uno de los pioneros del conservacionismo en el país y Edgard Roquette Pinto (1884-1954) forense, profesor, escritor, antropólogo, etnólogo y ensayista brasileño y considerado el padre de la radiodifusión en Brasil; asimismo, énfasis en la creación de revistas y establecimiento de métodos, prácticas científico y temas de estudio.

\footnotetext{
7 FIOCRUZ, "A Biologia Militante: Práticas Políticas e Divulgação Científica Na Era Vargas," Casa de Oswaldo Cruz, 2011, http://www.coc.fiocruz.br/index.php/pt/todas-as-noticias/909-a-biologia-militante-praticas-politicas-e-divulgacao-cientifica-na-era-vargas2\#.Xo40z5lv-Uk.

${ }^{8}$ Duarte, Activist Biology: The National Museum, Politics, and Nation Building in Brazil.

9 lbid.

10 Somos UFMG, "Regina Horta Duarte," n.d., http://somos.ufmg.br/professor/regina-horta-duarte.
} 
Aunque es un libro de corta extensión, presenta información robusta y sólida respaldada por una extensa y sólida investigación documental, la investigación se centra en la constitución del Museo Nacional como un espacio privilegiado para el conocimiento biológico y un lugar de acción relevante para los científicos en las décadas de 1920 y 1940. El libro se compone de tres capítulos: "Activist Biology", "A Miniature Of The Fatherland", "The Making Of A Biologist", Regina H. Duarte comienza el libro describiendo los procesos de consolidación de la biología como un campo diferente de La historia natural, además, ser una ciencia que produce nuevos conocimientos mediante la incorporación de nuevas técnicas. Para Brasil, en ese momento el conocimiento biológico todavía estaba muy fragmentado, contradictorio y como Regina H. Duarte menciona, entremezclado, lo cual, fue un aspecto importante para el desarrollo de esta investigación en biomedicina y agricultura, siendo objeto de estudio las enfermedades y plagas de la época e hizo tornar este campo de la ciencia, un espectro inseparable de la política de la época, aquí la autora presenta y trata de demostrar cómo entender la relación entre este campo y la dimensión política en Brasil.

Regina H. Duarte también presenta cómo la biología era un campo de conocimiento específico y cómo se estableció para resolver problemas políticos, especialmente después del surgimiento de la población como una cuestión de interés nacional, incluidos los principales investigadores Roquette-Pinto, Mello Leitão y Sampaio, autoridades del Museo Nacional, que desempeñaron un papel importante en la regulación y preservación del patrimonio de la flora y la fauna, en este sentido, la historiadora destaca los éxitos y frustraciones de los científicos. También describe las concepciones, proyectos e investigaciones biológicas de estos científicos, articulados con la concepción del Estado para desarrollar la nación, para fortalecer los proyectos orgánicos y cooperativos del gobierno de Vargas, así como sus proyectos e iniciativas de difusión del conocimiento.

Lo anterior sirve como escenario y establece una conexión con el segundo capítulo. En el cual, la historiadora examina cómo a lo largo de la historia, el Museo Nacional se ha convertido en un lugar para difundir conocimientos y prácticas 
esenciales para la población, argumentando que la educación era el medio por el cual, las personas podían entender el conocimiento científico y ser abrazados por la nación como pueblo. En otras palabras, como los biólogos crearon una diversidad de medios para difundir la idea de preservar la naturaleza del país. Según Regina H. Duarte, aprovecharon la radio, el cine, los periódicos y la Revista Nacional de Educación como medios promotores. También, adecuado para la época, el Museo Nacional prestó sus instalaciones para ofrecer lecciones prácticas con niños y jóvenes y acceso a su sala de exposiciones de antropología. Regina Horta Duarte también demuestra cómo el Museo Nacional, diseñado para ser lo que llamó la "miniatura da pátria", incorporó la función pedagógica, considerada central en este contexto para el conocimiento de la biodiversidad del país y para crear una sensación de patriotismo.

A pesar de todo este esplendor reflejado, Regina H. Duarte señala cómo algunos proyectos en el museo no siempre tuvieron el éxito esperado, como el proyecto de la revista nacional que, en 1934, creó el departamento de propaganda y difusión cultural. Sin embargo, en la década 1930 encontraron apoyo para sus proyectos que vincularon la naturaleza y la educación, el nombramiento de Gustavo Capanema como Ministro de Educación y Salud representaron algunos cambios, caracterizados principalmente por la reanudación de las campañas de salud, por el comienzo de estudios destinados a la creación de la Universidad de Brasil, asimismo, no permaneció ajeno al gran debate en los círculos culturales y políticos del país, sobre el significado y la orientación del sistema educativo brasileño. En este punto, "Activits biology" puede dialogar con diferentes obras como "Capanema: A história do ministro da Educação que atraiu intelectuais, tentou controlar o poder e sobreviveu à Era Vargas"11 de Fabio Silvestre Cardoso y "Moderno e Brasileiro: a história de uma nova linguagem na arquitectura (1930-60)"12 de Lauro Cavalcanti, donde presentan las diferentes decisiones que influenciaron de forma decisiva las características de la cultura brasileira como la conocemos en la actualidad, las cuales, fueron retratadas por Regina H. Duarte en este libro. Con base en lo anterior, el grupo proyecta

\footnotetext{
${ }^{11}$ Fábio Silvestre Cardoso, Capanema: A História Do Ministro Da Educação Que Atraiu Intelectuais, Tentou Controlar o Poder e Sobreviveu à Era Vargas (Rio de Janeiro: Record Editora, 2019).

12 Lauro Cavalcanti, Moderno e Brasileiro: A História de Uma Nova Linguagem Na Arquitectura (1930-60) (Rio de Janeiro: Jorge Zahar Editora, 2006).
} 
alrededor del espacio perdido del Museo Nacional otros temas en el horizonte de la política pública, como pensar en las nuevas directrices de la Universidad. La autora aclara que el grupo alrededor del Museo Nacional estaba inmerso en un "juego político" inherente a los desarrollos científicos prácticos que se desarrollan en este contexto. Al final de este capítulo, el autor examina la documentación de la Colección Brasiliana y analiza las ideas de los tres científicos publicados en varios libros que produjeron, y cuyo tema central era la biología en Brasil.

El tercer capítulo del libro, se centra en la trayectoria de Mello Leitão y en cómo se ha convertido en un experto en zoología y aracnología, desde su inserción en círculos científicos como la Academia de Ciencias y a través de redes de contacto establecidas con otros países americanos. En viajes e intercambio, además de centrarse en las estrategias que han convertido a este científico en un zoólogo de renombre internacional, lo cual, demuestra que el autor combina la excelencia con las preocupaciones académicas y educativas y el ejercicio de la biología como militancia. De igual forma, "Activist biology" puede dialogar con otra obra como "Proteção à natureza e identidade nacional no Brasil, anos 1920 - 1940"13 de José Luiz de Andrade Franco y José Augusto Drummond, donde se describe todo el papel fundamental que jugó Mello Leitão no solo para la ciencia en Brasil, sino también, para los diferentes países en Suramérica, ambos libros, muestran como este renombrado científico se preocupaba por el desarrollo de las investigaciones científicas en el campo de la biología en Brasil. Duarte nunca deja de mencionar que el Museo Nacional perdió fuerza al final de la era de Vargas como instrumento constituyente. La opinión pública y este discurso biológico se debilitaron de manera similar al símbolo de la identidad brasileira. Sin embargo, la biología se ha convertido en una ciencia importante en el escenario brasileño y en el espacio universitario del país.

El libro de Regina H. Duarte, tanto en su versión original, así como su versión en inglés goza de varias reseñas publicadas, que presentan diferentes opiniones y discusiones, que ayudan a enriquecer el eje central de esta obra, la biología activista. En este sentido, este libro ayuda a comprender los procesos para establecer cómo la

13 José Luiz de Andrade Franco and José Augusto Drummond, Proteção à Natureza e Identidade Nacional No Brasil, Anos 1920 - 1940 (Rio de Janeiro: SciELO - Editora FIOCRUZ, 2009). 
ciencia y la investigación dependen de los procesos políticos históricos, así como para comprender las articulaciones y los arreglos políticos que ciertos intelectuales hicieron en su época en el campo de la producción y en el juego de las relaciones con los poderes instituidos. Ciertamente, podemos decir que el libro muestra una pequeña parte de la historia y todos los diferentes desafíos y que los principales investigadores de la época tuvieron que superar para el establecimiento de la biología y la ciencia en Brasil. Regina H. Duarte intenta con su libro mostrar al lector el escenario, en el cual, se realiza el conocimiento creativo en las condiciones de posibilidades de cada investigación y cómo el papel del trabajo científico en la institución de las sociedades desempeñó un papel importante en el desarrollo de la sociedad brasileña.

\section{REFERENCIAS}

Cardoso, Fábio Silvestre. Capanema: A História Do Ministro Da Educação Que Atraiu Intelectuais, Tentou Controlar o Poder e Sobreviveu à Era Vargas. Rio de Janeiro: Record Editora, 2019.

Cavalcanti, Lauro. Moderno e Brasileiro: A História de Uma Nova Linguagem Na Arquitectura (1930-60). Rio de Janeiro: Jorge Zahar Editora, 2006.

Duarte, Regina Horta. A Biologia Militante: O Museu Nacional, Especialização Científica, Divulgação Do Conhecimento e Práticas Políticas No Brasil, 1926-1945. Belo Horizonte: Editora UFMG, 2010.

---. A Imagem Rebelde: A Trajetória Libertária de Avelino Fóscolo. São Paulo: Editora da Universidade Estadual de Campinas, 1991.

---. Activist Biology: The National Museum, Politics, and Nation Building in Brazil. Tucson, Arizona: University of Arizona Pres, 2016.

---. Noites Circenses: Espetáculos de Circo e Teatro Em Minas Gerais No Século XIX. São Paulo: Editora da Unicamp, 1995.

FIOCRUZ. “A Biologia Militante: Práticas Políticas e Divulgação Científica Na Era Vargas." Casa de Oswaldo Cruz, 2011. http://www.coc.fiocruz.br/index.php/pt/todas-as-noticias/909-a-biologiamilitante-praticas-politicas-e-divulgacao-cientifica-na-era-vargas-2\#.Xo40z5lvUk.

Franco, José Luiz de Andrade, and José Augusto Drummond. Proteção à Natureza e Identidade Nacional No Brasil, Anos 1920 - 1940. Rio de Janeiro: SciELO - Editora 
FIOCRUZ, 2009.

História, UFRJ-Instituto de. "Regina Horta Duarte, Biografia." Simpósio Diálogo BrasilEUA em História Ambiental, 2013. http://labhe.historia.ufrj.br/.

Somos UFMG. "Regina Horta Duarte," n.d. http://somos.ufmg.br/professor/reginahorta-duarte. 